\title{
EDITORIAL
}

\section{Yes to NO: the first studies on exhaled nitric oxide-driven asthma treatment}

\author{
J.C. de Jongste
}

$\mathbf{R}$ ecently, the first long-term studies examining the use of fractional exhaled nitric oxide (FeNO) to titrate steroid treatment in asthma were published $[1,2]$. Treatment decisions in asthma have always been made on the basis of symptoms, with or without taking peak flow into account. It is well established that, within an asthma population, both symptoms and airway obstruction correlate poorly with the presence and severity of airway inflammation. A strategy where inflammometry identifies the patient with airway inflammation would potentially prevent over treatment and allow for titration towards the lowest effective dose of steroids. It may also alert for under treatment or noncompliance. The validity of the concept of inflammometry-driven asthma management was supported by two earlier studies, both showing an important reduction in asthma exacerbations as a result of treatment strategies aimed at the reduction of bronchial hyperresponsiveness or sputum eosinophilia, both proxies for airway inflammation in asthma [3, 4]. These studies have had limited practical impact due to the invasive and timeconsuming nature of both hyperresponsiveness testing and sputum induction, and because the hyperresponsivenessdriven strategy led to an increased dose of inhaled steroids.

\section{FeNO AS AN INFLAMMOMETER IN ASTHMA}

The present introduction of FeNO measurements in clinical practise has been preceded by well over 1,000 published studies on FeNO in the past 15 yrs. These have established the genetic and molecular basis of nitric oxide (NO) generation and metabolism in the airways, the physiological and pathophysiological importance of NO in several respiratory diseases, and a number of confounding factors. FeNO can be measured within seconds with minimal discomfort and no risk to the patient. Routine methodology can be used in children from the age of $\geqslant 4-5 \mathrm{yrs}$. The results are highly reproducible and immediately available, and the test can be repeated easily and as often as needed. The American Thoracic Society and the European Respiratory Society have both published and updated guidelines for standardised measurement of $\mathrm{FeNO}$, and normal reference values with the recommended techniques are available $[5,6]$.

From the very beginning, it was clear that FeNO could be developed for clinical purposes. Abnormalities of FeNO have

Correspondence: J.C. de Jongste, Dept of Paediatrics, Division Paediatric Respiratory Medicine, Sophia Children's Hospital, Erasmus University Medical Center, PO Box 2060, 3000 CB Rotterdam, The Netherlands. Fax: 31104636811. E-mail: j.c.dejongste@erasmusmc.nl been described in an increasing number of respiratory and nonrespiratory disorders, and the role of FeNO in asthma has been extensively studied. It is now well established that FeNO is the first bedside test that indirectly assesses eosinophilic airway inflammation in the bronchial mucosa in asthmatics. Allergic asthmatics have high FeNO, which shows a rapid, dose-dependent response to corticosteroids. Possible applications of FeNO include diagnosis of asthma, monitoring of treatment response and treatment compliance, steroid dose titration, prediction of exacerbation or relapse, and screening for asthma.

\section{THE FIRST LONGITUDINAL STUDIES OF FeNO-DRIVEN ASTHMA TREATMENT}

SMITH et al. [1] addressed the question of whether FeNO measurements could be helpful in downtitrating inhaled steroids in adult asthmatics. A total of 92 subjects completed a dose titration phase followed by a 1-yr follow-up, while their asthma was treated according to their $\mathrm{FeNO}$, or Global Initiative for Asthma (GINA) guidelines [1]. After a run-in period where patients used 500 or $750 \mu \mathrm{g}$ of fluticasone q.d., their steroid dose was downtitrated at 4-weekly intervals in case their FeNO was low ( $<15 \mathrm{ppb}$, corresponding to $<35 \mathrm{ppb}$ with standard methodology). In the parallel control group, steroid downtitration followed when, during the past week, the patients reported one or no night-time awakenings, a mean peak flow amplitude of $<20 \%$, bronchodilator use less than four times on 1 or 2 days, and/or a forced expiratory volume in one second (FEV1) $>90 \%$. The optimal dose was fixed at a level one step higher, as where loss of control was seen. The study had a single-blind design. In the following year, patients were kept on this optimal dose, and stepped up in case of loss of control as defined above. Downtitration below the dose that produced control in the initial phase was not possible, i.e. the lower dose limit was fixed.

The results confirmed the null hypothesis, as the primary endpoint (exacerbations) showed no significant difference between the groups. However, at the end, the FeNO group used a significantly lower steroid dose $(45 \%)$ than the control group and, nevertheless, had at least the same level of asthma control by all other endpoints. The dose difference between the study arms was explained by an increased steroid dose in the control group rather than a reduced dose in the FeNO group. This may be the result of the study design, where the control group stepped up the steroid dose on the basis of any of five criteria, whereas in the FeNO group only FeNO determined the steroid dose. This imbalance might have caused more dose increments in the control group, perhaps resulting 
in over-treatment. SMITH et al. [1] argue that this algorithm corresponds to present GINA guidelines and that these, if rigorously applied, would indeed suffer from the same problem. In practise, however, these guidelines are not followed rigorously, to use a euphemism. Another point of concern is that after a downtitration phase, the steroid dose was fixed for the next 12 months, with only a possibility for dose increments in case of loss of asthma control. It might well be that during this year, the steroid dose could have been reduced further, and that FeNO monitoring could have identified these subjects better than conventional monitoring. This possible benefit was not taken into account. Remarkably, FeNO and induced sputum eosinophils did not differ at the end of the study between strategies and were low. This can again be explained by possible over-treatment of the control group.

The paediatric study by PIJNENBURG et al. [2] also used FeNO to adapt the steroid dose, but followed a protocol that was close to normal asthma management. Children diagnosed with allergic asthma were followed for $1 \mathrm{yr}$, used their own medication and were seen five times with 3-month intervals. At each visit their FeNO was measured and symptom diaries reviewed. Steroid doses were adapted on the basis of FeNO or symptoms, while both patients and doctors were unaware of group allocation. For ethical reasons, symptoms could overrule FeNO in case the symptom scores were high; in that case no dose reduction took place if FeNO was low. In all other situations, FeNO determined steroid dose in the FeNO group, with a cut-off point of $30 \mathrm{ppb}$, similar to that in the study by SMith et al. [1]. PIjnenburG et al. [2] hypothesised that FeNO would identify those children who were actually over-treated and could safely taper or stop their treatment, thereby targeting steroids to those children whose disease is due to chronic airway inflammation. The primary endpoint was a cumulative steroid dose over the whole year. Secondary endpoints included symptom scores, spirometry and bronchial responsiveness to methacholine. Surprisingly, no effect on steroid dose was found; both the FeNO and control group had the same cumulative steroid dose after 1 yr. However, a striking 2.5-doubling dose improvement in hyperresponsiveness was seen in favour of the FeNO group. Also, FEV1 improved significantly in the FeNO group, but started from a lower baseline and the differences in FEV1 were not significant between the groups. Unlike the study by SмIтH et al. [1], this study showed a significant difference in FeNO between the groups at the end, with elevated levels in the control group indicative of worse inflammation after $1 \mathrm{yr}$ of steroid dose titration on the basis of symptoms. Severe exacerbations, defined by the need for a course of oral prednisone, were more frequent in the control group (18 versus eight in the FeNO group), but the study was not powered to detect significant differences in severe exacerbations. Pijnenburg et al. [2] conclude that 3-monthly FeNO monitoring leads to a major reduction of hyperresponsiveness without the need for more steroids, and reduces airway inflammation.

\section{OTHER RECENT FINDINGS ON CLINICAL APPLICATION OF FeNO}

Several other recent studies have described applications of FeNO that may be helpful for daily practise and show positive results. Two studies have shown that FeNO predicts loss of asthma control or relapse after tapering the dose, or stopping steroids $[7,8]$. These studies did not address whether or not it is actually possible to prevent relapses by adapting treatment based on FeNO. Another study found FeNO levels a good predictor of clinical response to inhaled steroids in steroidnaive adults and children with chronic respiratory symptoms not typical for asthma [9].

\section{INTRODUCTION OF FENO INTO CLINICAL PRACTICE}

Long-term studies clearly show the feasibility of FeNO monitoring in paediatric and adult clinical practice and are suggestive of significant benefit of even infrequent monitoring of FeNO in asthmatics. Doctors and patients both showed good compliance with the protocol, indicating that FeNO-driven treatment was well accepted. This is certainly encouraging for adopting FeNO assessment in daily practice. However, there are some questions left. The study results entirely depend on the cut-off levels of FeNO, symptoms and other parameters of asthma control. In the absence of previous studies, the different authors have chosen their algorithms more or less arbitrarily and it is unclear what the effect of other cut-off levels or alternative dosing schedules would have been. Would, for instance, the difference in steroid dose as reported by SMITH et al. [1] no longer be seen with higher symptom cut-off levels in the control group? Other ways of dealing with FeNO are still unexplored, for instance the use of personal best values. What would be the effect of more frequent monitoring? FeNO not only reflects the inflammatory process in asthma, but also a direct effect of steroids on $\mathrm{NO}$ generation, and effects of confounders, including viral infections. Hence, a fall in FeNO does not necessarily mean that the inflammation is well treated, but may merely signify that a certain dose of the drug has reached the airways. This by itself is useful information, as compliance with treatment and bad inhaler technique are probably the main factors standing in the way of good asthma control in the majority of patients. However, it also raises the question of whether steroid downtitration is the proper response to, for example, a quick normalisation of FeNO within a couple of days. Now that the equipment for FeNO home monitoring has started becoming commercially available, studies are needed to tackle the issue of frequent monitoring.

\section{THINGS TO KEEP IN MIND WHEN USING FeNO}

Clearly, FeNO cannot be expected to be the perfect inflammometer. Its pitfalls and limitations should be realised. An important question is whether the recommended steroid dose steps are indeed effective in reducing high FeNO. Earlier observations in children suggest that doubling the steroid dose has only limited effects on FeNO in the majority of asthmatic children with elevated FeNO, despite conventional doses of inhaled steroids, and the mechanism behind this observation is not clear [10]. Perhaps, FeNO monitoring may be more useful for tapering than for stepping up steroids, and this should be resolved in future studies.

At group level, the benefits of FeNO monitoring seem clear. However, individual data may be puzzling and seem to suggest heterogeneity in the FeNO response to steroids. Individuals may respond well, with rapid normalisation of FeNO, or may show a partial response, with FeNO levels 
remaining elevated despite further increases of the steroid dose. Occasionally, very little change in FeNO is seen. This may be due to noncompliance or faulty inhaler technique, but there may also be genetic heterogeneity or different types of inflammation involved, e.g. neutrophilic versus predominantly eosinophilic, and this needs to be sorted out.

Most of the studies on FeNO in asthma are concerned with allergic patients and it has been argued that FeNO should only be used to manage allergic asthmatics. In the study by SMITH et al. [1] allergy was not an inclusion criterium. A total of 16 out of 96 randomised patients were nonallergic and these showed a heterogeneous picture. Some of the patients developed a rise in FeNO, symptoms and sputum eosinophilia upon downtitration of their steroid treatment, while in others the steroids could be downtitrated to placebo without problems (A.D. Smith and D.R. Taylor, Respiratory Research Unit, Dept of Medicine, Dunedin School of Medicine, University of Otago, Dunedin, New Zealand, personal communication). PIJNENBURG et al. [2] studied allergic asthmatics only. On the basis of these results, it is premature to assume that FeNO is only useful in allergic asthma and this should be addressed by future studies powered to answer this question.

\section{CONCLUSIONS}

In summary, the new studies are ground breaking as they are highly suggestive of long-term benefit of FeNO inflammometry in asthma management. The bottom line is that FeNO inflammometry is an easy test which is helpful to target steroid treatment, to reduce steroids where possible, and to provide significant benefits in terms of less hyperresponsiveness and less inflammation without leading to overall need for more steroids at the group level. On the basis of these findings, FeNO offers more for day-to-day asthma management than any of the conventional lung function tests. Now FeNO analysers have become available for office and ambulatory measurements and become more affordable, there is little standing in the way of a widespread use in clinical practice. Not only the applications in asthma management warrant the introduction of FeNO inflammometry into clinical practice, but also a number of other indications, including diagnosis of asthma, prediction of loss of asthma control and screening for primary ciliary dyskinesia.

Now the benefits are becoming clear, the momentum is there to start the procedures that will lead to appropriate incorporation of fractional exhaled nitric oxide measurements into national reimbursement codes, paving the way for inflammometrydriven asthma management.

\section{REFERENCES}

1 Smith AD, Cowan JO, Brasset KP, Herbison P, Taylor DR. Use of exhaled nitric oxide measurements to guide treatment in chronic asthma. N Engl J Med 2005; 352: 2163-2173.

2 Pijnenburg MW, Bakker EM, Hop WC, de Jongste JC. Titrating steroids on exhaled nitric oxide in asthmatic children: a randomized controlled trial. Am J Respir Crit Care Med 2005; Epub ahead of print.

3 Sont JK, Willems LN, Bel EH, van Krieken JH, Vandenbroucke JP, Sterk PJ. Clinical control and histopathologic outcome of asthma when using airway hyperresponsiveness as an additional guide to long-term treatment. The AMPUL Study Group. Am J Respir Crit Care Med 1999; 159: 1043-1051.

4 Green RH, Brightling CE, McKenna S, et al. Asthma exacerbations and sputum eosinophil counts: a randomised controlled trial. Lancet 2002; 360: 1715-1721.

5 American Thoracic Society. ATS/ERS Recommendations for standardized procedures for the online and offline measurement of exhaled lower respiratory nitric oxide and nasal nitric oxide, 2005. Am J Respir Crit Care Med 2005; 171: 912-930.

6 Buchvald F, Baraldi E, Carraro S, et al. Measurements of exhaled nitric oxide in healthy subjects age 4 to 17 years. $J$ Allergy Clin Immunol 2005; 115: 1130-1136.

7 Zacharasiewicz A, Wilson N, Lex C, et al. Clinical use of noninvasive measurements of airway inflammation in steroid reduction in children. Am J Respir Crit Care Med 2005; 171: 1077-1082.

8 Pijnenburg MW, Hofhuis W, Hop WC, de Jongste JC. Exhaled nitric oxide predicts asthma relapse in children with clinical asthma remission. Thorax 2005; 60: 215-218.

9 Smith AD, Cowan JO, Brasset KP, et al. Exhaled nitric oxide: a predictor of steroid response. Am J Respir Crit Care Med 2005; (In press).

10 Pijnenburg MWH, Bakker EM, Lever S, Hop WC, de Jongste JC. High fractional concentration of nitric oxide in exhaled air despite steroid treatment in asthmatic children. Clin Exp Allergy 2005; 37: 920-925. 